\title{
A influência do evento El Niño - Oscilação Sul e Atlântico Equatorial na precipitação sobre as regiões norte e nordeste da América do Sul
}

\author{
Rosimeire ARAÚJO Gonzalez ${ }^{1}$, Rita Valéria ANDREOLI², Luiz Antonio CANDIDO³, Mary Toshie \\ KAYANO ${ }^{4}$, Rodrigo Augusto Ferreira de SOUZA ${ }^{5}$
}

\begin{abstract}
RESUMO
Os impactos de eventos anômalos no oceano Pacífico associados ao El Niño-Oscilação Sul (ENOS) na precipitação da regiáo norte e nordeste da América do Sul foram avaliados para o período de 1900 a 2007, fazendo-se uso de análise de composiçôes. Os eventos El Niño (La Niña) no Pacífico que juntamente com um Modo Equatorial no Atlântico (MEA) frio (quente) formam um gradiente interbacias entre o Pacífico e Atlântico foram analisados considerando, separadamente, aqueles para os quais o gradiente se forma na fase inicial do ENOS daqueles em que o gradiente se forma na fase de decaimento do ENOS. Os resultados mostram que o padrão de precipitação na regiáo norte e nordeste da América do Sul é reforçado mediante a configuraçáo do gradiente interbacias durante a fase inicial do ENOS. Nesse caso, uma possível explicaçáo é que o MEA de sinal contrário ao ENOS durante sua fase inicial cria condiçôes favoráveis para o desenvolvimento de um gradiente inter-hemisférico no Atlântico Tropical atuando no mesmo sentido do gradiente interbacias, e colaborando para fortalecer o efeito do El Niño (La Niña) na precipitação. Por outro lado, para os eventos ENOS em que o gradiente se forma em sua fase de decaimento, o impacto na precipitaçáo é mais significativo na regiáo norte e centro-oeste da bacia. Uma possível explicação para essas diferenças está associada às mudanças que ocorrem na circulação atmosférica leste-oeste associada ao gradiente lesteoeste de anomalias da TSM. Os resultados deste estudo podem ser úteis, principalmente, para fins de monitoramento climático.
\end{abstract}

PALAVRAS-CHAVE: Temperatura da Superfície do Mar, chuva, Amazônia, gradiente interbacias

\section{Influence of El Niño-Southern Oscillation and Equatorial Atlantic on rainfall over northern and northeastern regions of South America.}

\begin{abstract}
The impacts of anomalous events in the Pacific Ocean associated with the El Niño - Southern Oscillation (ENSO) on rainfall over northern and northeastern regions of South America were evaluated for the period from 1900 to 2007 using composite analyses. The El Niño (La Niña) events in the Pacific, that together with a cold (warm) Atlantic Equatorial Mode (AEM) form an interbasin gradient between the Pacific and Atlantic were analyzed considering separately those for which the gradient forms during the ENSO onset phase from those for which the gradient forms during the ENSO demise phase. The results show that the rainfall pattern over the northern and northeastern region of South America is reinforced under an interbasin gradient during the initial phase of the ENSO event. In this case, a possible explanation is that the AEM with opposite sign of the ENSO event in its onset stage creates favorable conditions for the development of an interhemispheric gradient in the Tropical Atlantic acting in the same direction of the interbasin gradient, and collaborating to reinforce the El Niño (La Niña) effect on the precipitation. On the other hand, for ENSO events for which the interbasin gradient forms in the demise stage, the impact on the precipitation is more significative in the northern and central-western regions of the basin. A possible explanation for these differences is linked to the alterations in the east-west atmospheric circulation associated with the east-west gradient of the SST anomalies. The result of this study might be useful mainly for climate monitoring purposes.
\end{abstract} KEYWORDS: Sea Surface Temperature, rainfall, Amazon, interbasin gradient

\footnotetext{
1 Programa de Pós-Graduação em Clima e Ambiente (CLIAMB, INPA/UEA). Av. André Araújo, 2936, Campus II, Aleixo, 69060-001, Manaus, AM, Brasil. projetorose@gmail.com 2,5 Universidade do Estado do Amazonas, Escola Superior de Tecnologia, Coordenação de Meteorologia. Av. Darcy Vargas, 1200, Parque 10 de Novembro, 69065-020, Manaus, AM, Brasil.2rasouza@uea.edu.br, 5rafsouza@uea.edu.br

3 Instituto Nacional de Pesquisas da Amazônia-INPA, Coordenação de Dinâmica Ambiental. Av. André Araújo, 2936, Campus II, Aleixo, 69060-001, Manaus, AM, Brasil. Icandido@inpa.gov.br

4 Instituto Nacional de Pesquisas Espaciais, Centro de Previsão de Tempo e Estudos Climáticos, Divisão de Modelagem e Desenvolvimento. Av. dos Astronautas, 1758, 12227-010, São José dos Campos, SP, Brasil. mary.kayano@cptec.inpe.br
} 


\section{INTRODUÇÃO}

Estudos com enfoque na variabilidade interanual da precipitação nas regiôes norte e nordeste da América do Sul têm associado essa variabilidade ao fenômeno El NiñoOscilação Sul (ENOS) (Kayano e Moura 1986; Marengo 1992; entre outros). Anomalias positivas (negativas) da temperatura da superfície do mar (TSM) no Oceano Pacífico associadas aos episódios El Niño (La Niña) produzem circulaçóes anômalas de grande escala na atmosfera, gerando impactos significativos no clima da regiâo do Atlântico e da América do Sul (Aceituno 1988; Grimm et al. 2000; Grimm 2004). Neste mesmo contexto, Marengo (2006) observou, para o período de 1979-2000, reduções na precipitação, no escoamento e na convergência de umidade em anos de El Niño, e aumentos destas variáveis em anos de La Niña.

Por outro lado, (Yoon e Zeng 2010) constataram que apenas uma fração da variabilidade da precipitação na Amazônia pode ser explicada pelo ENOS, e que esta relaçấo é mais atuante somente durante a estaçáo chuvosa. Os autores sugerem que a influência do Atlântico Tropical (AT) Norte (ATN) na variabilidade climática na regiáo Amazônica pode ser comparável às mais conhecidas teleconexões com o ENOS do oceano Pacífico, concordando com estudos anteriores que mostraram que as variações de precipitação da Amazônia também estão relacionadas às TSM no AT (Liebmann e Marengo 2001; Marengo et al. 2008).

Assim, estudos que examinam a variabilidade interanual de precipitação nas regiôes norte e nordeste da América do Sul devem levar em conta as variaçóes interanuais de TSM associadas ao fenômeno ENOS e as que ocorrem no AT, bem como suas relaçôes. Objeto de vários estudos têm sido os efeitos das variaçóes da TSM do Pacífico tropical em outras regiôes oceânicas (Curtis e Hastenrath 1995; Enfield e Mayer 1997; Giannini et al. 2001). Nesses estudos está claro que o ENOS influencia tanto o ATN quanto o AT sul (ATS). Porém, a influência do ENOS no Atlântico Equatorial é menos clara (Zebiak 1993; Enfield e Mayer 1997), e pode ocorrer no sentido oposto.

De fato, mudanças na teleconexão entre os oceanos Atlântico e Pacífico vêm sendo notadas. Rodríguez-Fonseca et al. (2009) encontraram evidências que desde o final da década de 60 os eventos de ENOS no Pacífico são precedidos por eventos de sinal oposto nas anomalias TSM (ATSM) do Atlântico Equatorial. O mecanismo proposto para essa relação é que um evento de anomalia positiva (negativa) no Atlântico Equatorial intensifica uma circulação de Walker anômala com movimentos ascendentes (descendentes) sobre o Atlântico Equatorial e descendente (ascendente) sobre o Pacífico equatorial leste (Wang 2006; RodriguezFonseca et al. 2009; Kayano et al. 2011). Esse mecanismo induz processos oceânicos que favorecem o afloramento (afundamento) da termoclina, o que contribui para que a região do Pacífico equatorial leste se torne ainda mais fria (quente), intensificando-se a La Niña (o El Niño) existente.

Kayano et al. (2011), analisaram a evolução temporal das ATSM relacionadas ao ENOS que ocorreram após eventos quentes/frios no Atlântico Equatorial e aqueles que ocorrem independentemente das condiçóes do Atlântico Equatorial. Seus resultados mostram diferentes padrôes de evoluçấo das anomalias de TSM e de pressão ao nível do mar (PNM), principalmente em relaçấo às teleconexôes associadas ao ENOS com efeitos na precipitação.

Do acima exposto, os resultados anteriores sugerem a existência da relação interbacias. No entanto há discrepâncias sobre a questão da precedência. Alguns autores sugerem que o Pacífico Tropical tem uma significativa influência no AT (Enfield e Mayer 1997; Latif e Grotzer 2000), enquanto recentes estudos têm mostrado uma associação reversa, de modo que um evento quente (frio) no Atlântico Equatorial pode ser seguido de um evento frio (quente) no Pacífico Tropical (Melice Servain 2003; Rodriguez-Fonseca et al. 2009; Kayano et al. 2011). O presente trabalho re-examina a relaçáo entre o ENOS e evento quente (frio) no Atlântico Equatorial estabelecendo as sequências de padróes anômalos de TSM e precipitação no norte da América do Sul considerando que casos de ENOS podem preceder ou suceder eventos anômalos e de sinais contrários no Atlântico Equatorial. Vale ressaltar que a abordagem aqui é similar a de Kayano et al. (2011). Porém, na presente análise, um índice mais simples foi usado para definir os eventos quentes e frios do AT, os eventos selecionados de ENOS foram os mais fortes, e o enfoque na precipitação é para as estaçóes seca e chuvosa da regiấo Amazônica. Estes aspectos facilitam a aplicação dos resultados para monitoramento climático, bem como para simulaçóes climáticas futuras.

\section{MATERIAL E MÉTODOS}

Os dados utilizados para definir os padróes dominantes de variabilidade de TSM nos oceanos Pacífico e Attântico sáo os campos globais de TSM mensais, que consistem de séries temporais reconstruídas por Smith et al. (2008) para o período de 1900 a 2008 numa grade com uma resolução espacial de $2^{\circ}$ de latitude por $2^{\circ}$ de longitude. Em adição são usadas séries mensais de Pressão ao Nível do Mar (PNM) em uma resolução espacial de $5^{\circ}$ de latitude por $5^{\circ}$ de longitude, para o período de 1901 a 2004 obtidas a partir do site do "Met Office Hadley Center" (Allan e Ansell 2006), e séries de precipitaçôes mensais em uma resoluçấo espacial de $1^{\circ}$ de latitude por $1^{\circ}$ de longitude derivadas do "Global Precipitation Climatology Center (GPCC)” (Beck et al. 2005; Rudolf e Rubel 2005). As séries de precipitaçáo sáo selecionadas para o período de 1901-2007 na área delimitada por $5^{\circ} \mathrm{N}, 20^{\circ} \mathrm{S}$, 
$90^{\circ} \mathrm{W}$ e $30^{\circ} \mathrm{W}$, a qual engloba as regiôes norte e nordeste da América do Sul. Estes dados foram gerados por um sistema de análises operacional o qual inclui: integraçâo de dados a partir de diferentes fontes, controle de qualidade, e o cálculo da precipitação média em área em cada grade de $1^{\circ}$ por $1^{\circ}$ (Rudolf e Rubel 2005). Embora erros possam existir neste conjunto de dados devido a deficiências no processo de medição ou a representatividade espacial, o mesmo contém dados de precipitaçáo em pontos de grade com grande fidelidade às medidas locais, e já foi usado em diversos estudos anteriores, que avaliaram a variabilidade de precipitação sobre a região de estudo, dentre eles: Jury (2009) e Kayano et al. (2011).

As ATSM mensais foram calculadas removendo-se o ciclo anual usando como base a climatologia mensal do período de 1901 a 2007. Em seguida a tendência linear de longo prazo foi excluída em cada ponto de grade. Os eventos ENOS são selecionados através do índice da regiáo do Niño-3, definida por Trenberth et al. (1997), como sendo a média espacial das ATSM sobre a regiáo delimitada por $4^{\circ} \mathrm{N}, 4^{\circ} \mathrm{S}, 150^{\circ} \mathrm{W}$ e $90^{\circ} \mathrm{W}$. Valores trimestrais do Niño-3 para DJF (dezembrojaneiro-fevereiro) maiores que $0,9^{\circ} \mathrm{C}$ (menores que $-0,7^{\circ} \mathrm{C}$ ) identificam o ano que corresponde a dezembro como sendo o ano inicial [ano (0)] do evento quente (frio) do ENOS. O ano seguinte $[a n o(+1)]$ se refere à fase madura do evento. $\mathrm{O}$ limiar para os eventos frios é menor para refletir a assimetria na distribuição do aquecimento/resfriamento da regiấo do Niño-3. Esta metodologia é similar à utilizada por Andreoli e Kayano (2006), e seleciona eventos mais intensos.

O índice mensal que descreve as variaçôes de TSM no setor equatorial do AT, que representa a regiáo do Modo Equatorial do Atlântico (MEA), é obtido calculando-se a média das ATSMs na regiáo definida por Zebiak (1993) delimitada em $4^{\circ} \mathrm{S}, 4^{\circ} \mathrm{N}, 20^{\circ} \mathrm{W}$ e $0{ }^{\circ} \mathrm{W}$. Valores do índice do MEA médios para JJA maiores que $0,4^{\circ} \mathrm{C}$ (menores que $-0,4^{\circ} \mathrm{C}$ ) identificam os eventos MEA positivos (negativos) no Atlântico Equatorial, para os anos correspondentes às fases inicial (0) e de decaimento (+1) do ENOS.

Para separar, dentre os eventos que ocorrem no Pacífico aqueles que estiveram associados ao MEA, adota-se o seguinte procedimento:

1. fase inicial de evento quente (frio) no Pacífico associado ao evento MEA frio (quente), ou seja, índice do MEA inferiores a $-0.4{ }^{\circ} \mathrm{C}$ (superiores a $0,4^{\circ} \mathrm{C}$ ) ocorrendo no ano (0) de um evento ENOS. Estes eventos são definidos pelas siglas ATLN_EN e ATLP_LN.

2. fase madura do evento quente (frio) no Pacífico precedendo um evento frio (quente) no Atlântico Equatorial, ou seja, índice do MEA inferiores a $-0,4^{\circ} \mathrm{C}$ (superiores a $0,4^{\circ} \mathrm{C}$ ) ocorrendo no ano $(+1)$ do evento ENOS. Estes eventos sáo definidos pelas siglas EN_ATLN e LN_ATLP.
Em seguida, a análise de composição é utilizada para obter os campos espaciais trimestrais de ATSM e de anomalias de PNM a fim de ilustrar a evolução temporal dos eventos. O trimestre inicial do padrão de evoluçấo corresponde a $\operatorname{MAM}(0)$ e o trimestre final, a JJA(+1). No caso da precipitação, as análises são realizadas para os períodos de $\operatorname{DJF}(0), \operatorname{MAM}(+1)$ e JJA(+1). Anomalias mensais de PNM e precipitação foram obtidas considerando a climatologia para o período de 1901 a 2004 e 1901 a 2007, respectivamente. As séries de anomalias, em cada ponto de grade, são normalizadas pelo desvio padrão correspondentes às séries temporais de anomalias. Para obter a significância estatística desses campos aplicou-se o teste t de Student considerando o número de graus de liberdade como número de eventos selecionados.

\section{RESULTADOS}

A Tabela 1 lista os anos classificados como eventos El Niño/La Niña no Oceano Pacífico e aqueles que configuram um gradiente interbacias ocorrendo na fase inicial (de decaimento) de eventos de El Nińo denotados por ATLN EN (EN_ATLN), e eventos que configuram um gradiente interbacias ocorrendo na fase inicial (de decaimento) de eventos La Niña são denotados por ATLP_LN (LN_ATLP). Nesta Tabela, cada evento ENOS é indicado pelo ano que corresponde à fase inicial do evento. Análises da Tabela em termos de porcentagens mostram que $33 \%$, ou seja, sete dos 21 eventos de El Niño foram precedidos por um evento frio no Atlântico Equatorial (ATLN_EN). A mesma quantidade em termos percentuais foi encontrada para o caso de um evento frio no Atlântico equatorial ocorrendo após um El Niño (EN_ATLN). Em relação aos eventos de La Nińa, dos 26 eventos selecionados, seis foram precedidos por um evento quente no Atlântico Equatorial (ATLP_LN), totalizando $23 \%$ dos eventos, enquanto que sete eventos, ou ainda, $26 \%$ dos eventos precederam um evento quente no Atlântico Equatorial (LN_ATLP). Esses resultados são consistentes com os encontrados por Kayano et al. (2011), em relação aos eventos ENOS que são precedidos por eventos no Atlântico Equatorial. Ainda, em concordância com os resultados de Rodríguez-Fonseca et al. (2009), a conexão entre evento quente (frio) no Atlântico equatorial precedendo evento frio (quente) no Pacífico é mais evidente a partir do final da década de 60, quando se analisa o período de 1950 a 2000.

A fim de examinarmos as diferenças nos padrões de anomalias de TSM e PNM e suas relaçôes com precipitação nas regióes norte e nordeste da América, para os eventos selecionados na Tabela 1 , as composiçóes para essas variáveis foram obtidas e suas análises são apresentadas a seguir. 
Tabela 1 - Anos selecionados como eventos El Niño/La Niña no Oceano Pacífico. Anos que configuram um gradiente interbacias ocorrendo na fase inicial (de decaimento) de eventos de El Niño são denotados por ATLN EN (EN ATLN), e eventos que configuram um gradiente interbacias ocorrendo na fase inicial (de decaimento) de eventos La Niña são denotados por AT̄LP_LN (LN_ATLP).

\begin{tabular}{|c|c|c|c|c|c|}
\hline EL NIÑO & $\begin{array}{l}\text { ATLN_EN } \\
7 \text { Eventos } \\
(33 \%)\end{array}$ & $\begin{array}{l}\text { EN_ATLN } \\
7 \text { Eventos } \\
(33 \%)\end{array}$ & LA NIÑA & $\begin{array}{l}\text { ATLP_LN } \\
6 \text { Eventos } \\
(23 \%)\end{array}$ & $\begin{array}{l}\text { LN_ATLP } \\
7 \text { Eventos (26\%) }\end{array}$ \\
\hline 1902 & 1902 & & 1903 & & \\
\hline 1904 & 1904 & & 1908 & & 1908 \\
\hline 1905 & & 1905 & 1909 & 1909 & \\
\hline 1911 & 1911 & & 1910 & & \\
\hline 1913 & & 1913 & 1916 & & 1916 \\
\hline 1914 & 1914 & & 1917 & & \\
\hline 1918 & & 1918 & 1922 & & \\
\hline 1925 & & & 1924 & 1924 & 1924 \\
\hline 1930 & & & 1933 & & 1933 \\
\hline 1940 & & & 1938 & & 1938 \\
\hline 1957 & & 1957 & 1942 & & \\
\hline 1963 & & 1963 & 1944 & & \\
\hline 1965 & 1965 & & 1949 & & \\
\hline 1968 & & 1968 & 1950 & & \\
\hline 1972 & & & 1954 & & \\
\hline 1982 & 1982 & & 1955 & & \\
\hline 1986 & & & 1967 & & 1967 \\
\hline 1991 & & 1991 & 1970 & & \\
\hline 1994 & & & 1971 & & \\
\hline 1997 & 1997 & & 1973 & 1973 & \\
\hline \multirow[t]{6}{*}{2002} & & & 1975 & & \\
\hline & & & 1984 & 1984 & \\
\hline & & & 1988 & 1988 & \\
\hline & & & 1998 & & 1998 \\
\hline & & & 1999 & 1999 & \\
\hline & & & 2005 & & \\
\hline
\end{tabular}

\section{Fase positiva do ENOS e suas relações com a precipitação nas regiões norte e nordeste da América do Sul}

As Figuras 1 e 2 (painéis à esquerda, ATLN_EN) mostram os padróes espaciais de ATSM e anomalias de PNM associados ao desenvolvimento do gradiente interbacias, com a configuração de um evento frio no Atlântico equatorial ocorrendo na fase inicial de um evento de El Niño no Pacífico. Esse padrão mostra o fortalecimento de um evento El Niño com início em JJA(0). Nesse mesmo tempo, o gradiente interbacias de ATSM entre os oceanos Pacífico e Atlântico equatorial, está bem estabelecido. Anomalias negativas menos intensas persistem no Atlântico equatorial até $\mathrm{DJF}(0)$ quando o El Niño atinge sua fase madura. A partir de $\operatorname{DJF}(0)$ o El Niño começa a se enfraquecer, apesar de as ATSM positivas próximas à costa oeste da América do Sul persistirem até JJA(+1). No AT, o aquecimento no ATN, em resposta ao El Nińo, como mostrado em estudos anteriores (por ex. Enfield e Mayer 1997), não se estabelece por muito tempo. No ATS,
ATSM negativas voltam a se intensificar a partir de $\operatorname{MAM}(+1)$ e em JJA(+1) atingem a região equatorial, quando o gradiente interbacias é novamente estabelecido. Também, são notáveis no AT indicações de um fraco (anomalias não significativas) dipolo inter-hemisférico positivo em $\mathrm{MAM}(+1)$ e em JJA(+1).

Consistente com o padrão de ATSM durante o desenvolvimento do El Nińo em JJA(0), o padrão de anomalias de PNM caracteriza-se por um gradiente leste-oeste com anomalias positivas (negativas) significativas no Pacífico tropical oeste (leste) (Figura 2, painéis à esquerda). Anomalias positivas significativas de PNM também são notadas no Atlântico equatorial e ATN. O centro de anomalias positivas no AT intensifica nos passos de tempo seguinte e em $\operatorname{DJF}(0)$, estende-se desde a América do Sul até a África. $\operatorname{Em~MAM(+1),~}$ embora anomalias de TSM permaneçam na parte central e leste do Pacífico, o padrão de PNM se enfraquece. Pode-se inferir que associado a esse padrão anômalo de PNM uma circulação de Walker anômala se estabelece com subsidência de ar no Atlântico equatorial e parte leste da América do 

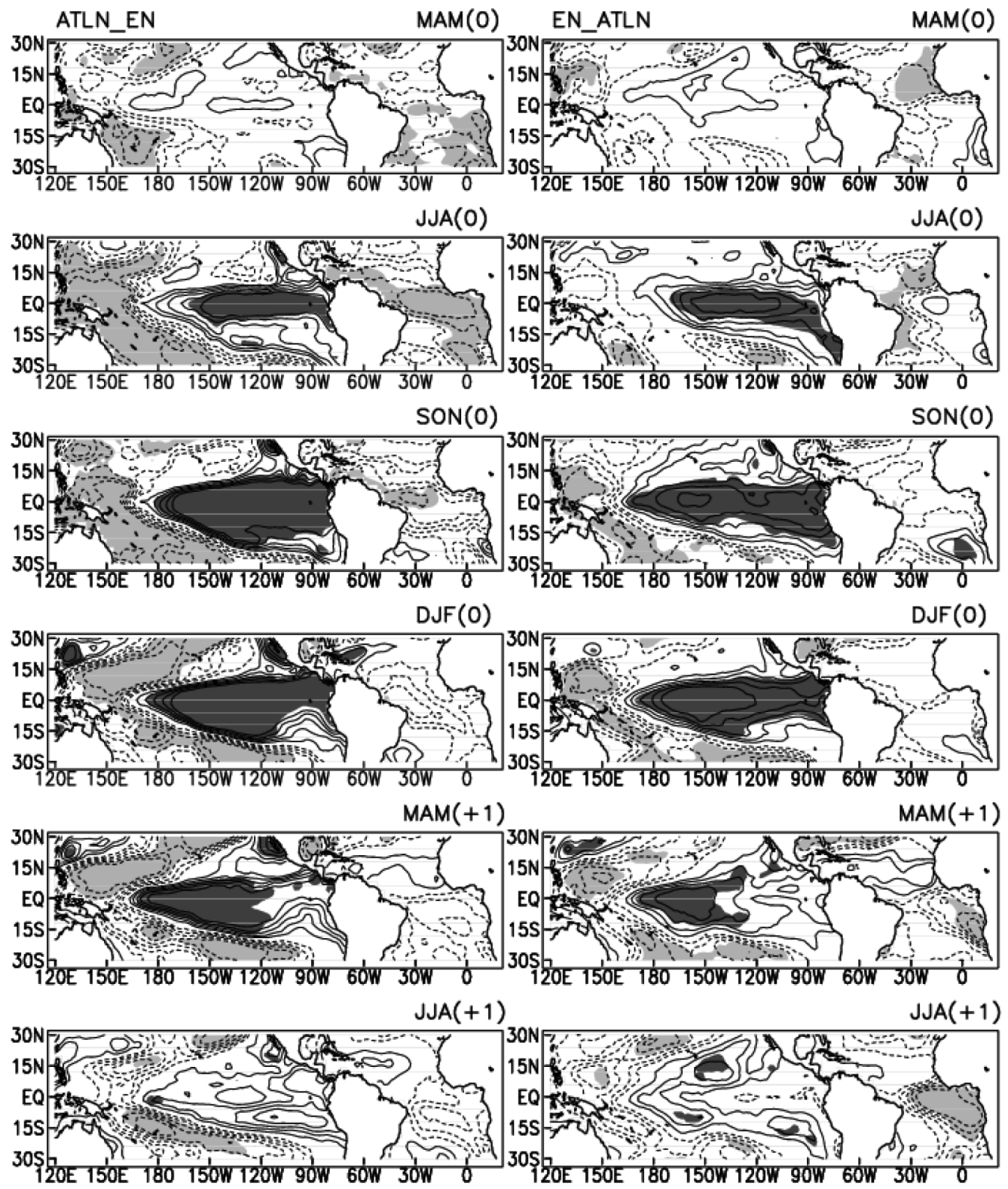

Figura 1 - Composições trimestrais de anomalias de TSM normalizadas a partir de MAM (0) a JJA (+1) para os eventos ATLN_EN e EN_ATLN. ContornoS contínuos (pontilhados) correspondem aos valores positivos (negativos). Intervalo de contorno é 0.2. Sombreados em escala de cinza claro (escuro) definem regiões com valores negativos (positivos) significativos ao nível de confiança de $95 \%$.

Sul e ascendência no Pacífico central e leste e costa oeste da América do Sul. No AT, consistente com o padrão de ATSM em MAM(+1) e JJA(+1), se estabelece um fraco dipolo interhemisférico negativo de anomalias de PNM.

Para o caso de MEA frio se estabelecendo na fase de decaimento do El Nino (Figura 1, painéis à direita), o evento El Niño inicia-se em JJA(0) e atinge máxima intensificação em $\operatorname{DJF}(0)$. Porém neste caso, as ATSM no Pacífico tropical estáo equatorialmente mais confinadas do que no caso descrito acima, em particular em SON(0). No AT, ATSM negativas no ATN e próximas à costa sudeste da América do Sul são notadas em JJA(0). Estas anomalias praticamente desaparecem a partir de $\operatorname{SON}(0)$. Vale notar que em $\operatorname{SON}(0)$ e $\mathrm{DJF}(0)$, há indicaçôes de ATSM positivas no ATS ao sul de $10^{\circ} \mathrm{S}$. Por outro lado, surgem ATSM positivas no ATN e negativas no ATS em resposta ao aquecimento do Pacífico em $\operatorname{MAM}(+1)$ concordando com estudos anteriores. Por exemplo, Enfield e Mayer (1997) mencionam a formaçáo desta estrutura dipolar no padrão de ATSM no AT durante o período de março a maio após um evento El Niño no Pacífico. As anomalias negativas 

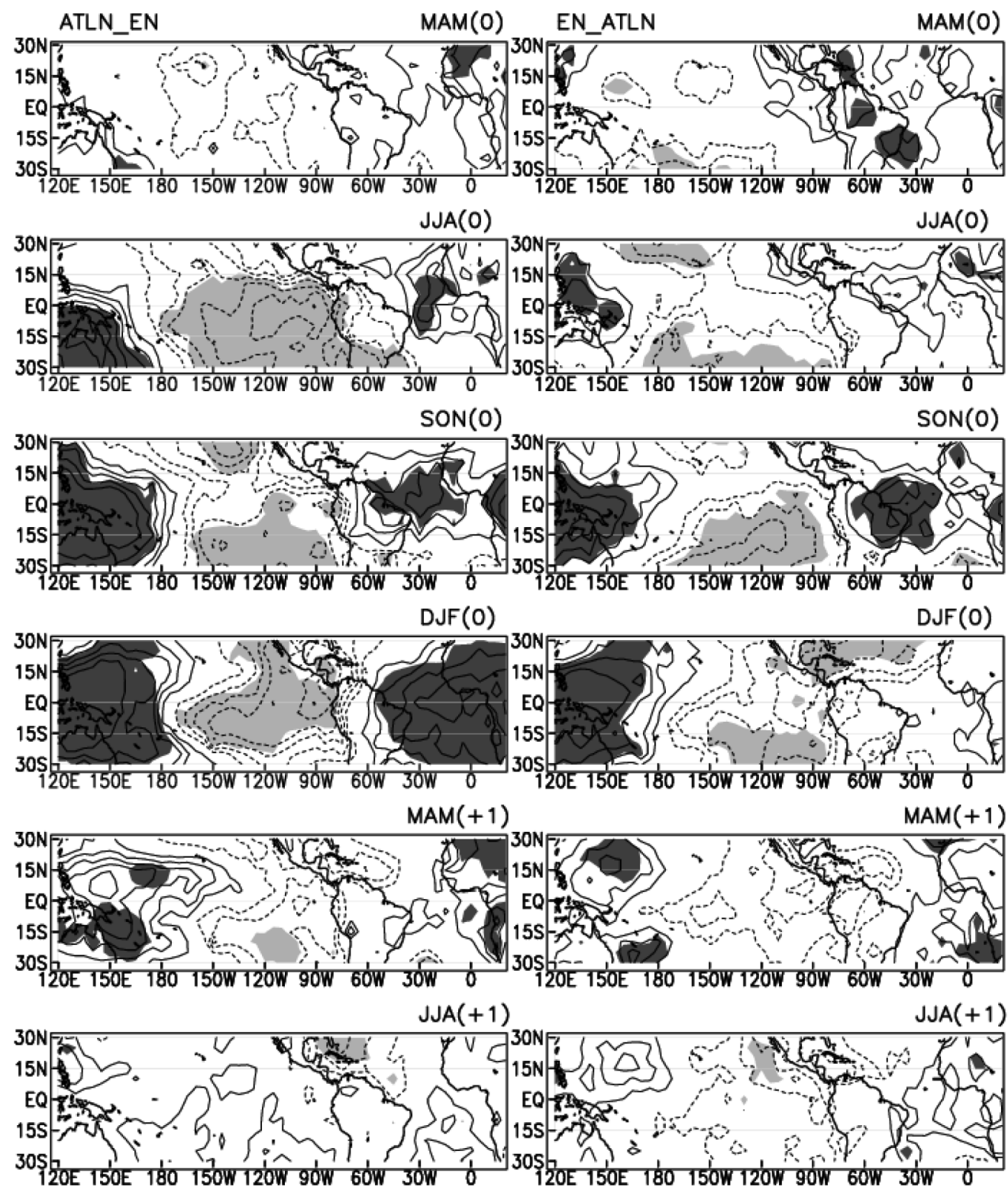

Figura 2 - Composições trimestrais de anomalias de PNM normalizadas a partir de MAM(0) a JJA(+1) para os eventos ATLN_EN e EN_ATLN. Contornos contínuos (pontilhados) correspondem aos valores positivos (negativos). Intervalo de contorno é 0.2. Sombreados em escala de cinza claro (escuro) definem regiões com valores negativos (positivos) significativos ao nível de confiança de $95 \%$.

no ATS e o El Nińo no Pacífico leste formam o gradiente interbacias em $\operatorname{MAM}(+1)$, que desaparece em $J J A(+1)$. Salientam-se em JJA(+1) as indicaçóes de ATSM positivas no ATN contornando o noroeste da América do Sul e no ATS próximas à costa leste do nordeste do Brasil.

Em relação aos padróes de anomalias de PNM, um gradiente leste-oeste anômalo com anomalias positivas na região da Austrália e negativas no Pacífico tropical leste é estabelecido em $\mathrm{SON}(0)$ e persiste até $\mathrm{DJF}(0)$ quando o El Niño atinge máxima intensificação (Figura 2, painéis à direita). $\mathrm{Em} \mathrm{SON}(0)$, anomalias positivas de $\mathrm{PNM}$ são notadas sobre a regiáo norte da América do Sul e oceano Atlântico adjacente. Esse centro de anomalias positivas praticamente desaparece em $\mathrm{DJF}(0)$, quando anomalias negativas de PNM são estabelecidas no ATN. O enfraquecimento das anomalias positivas de PNM é resultado da resposta atmosférica ao El Niño, conforme discutido por Enfield e Mayer (1997). Estes autores sugerem que a estrutura dipolar de TSM (PNM) no AT desenvolve-se durante a primavera boreal, seguida de um evento de El Nińo no Pacífico, conforme mostrado em 

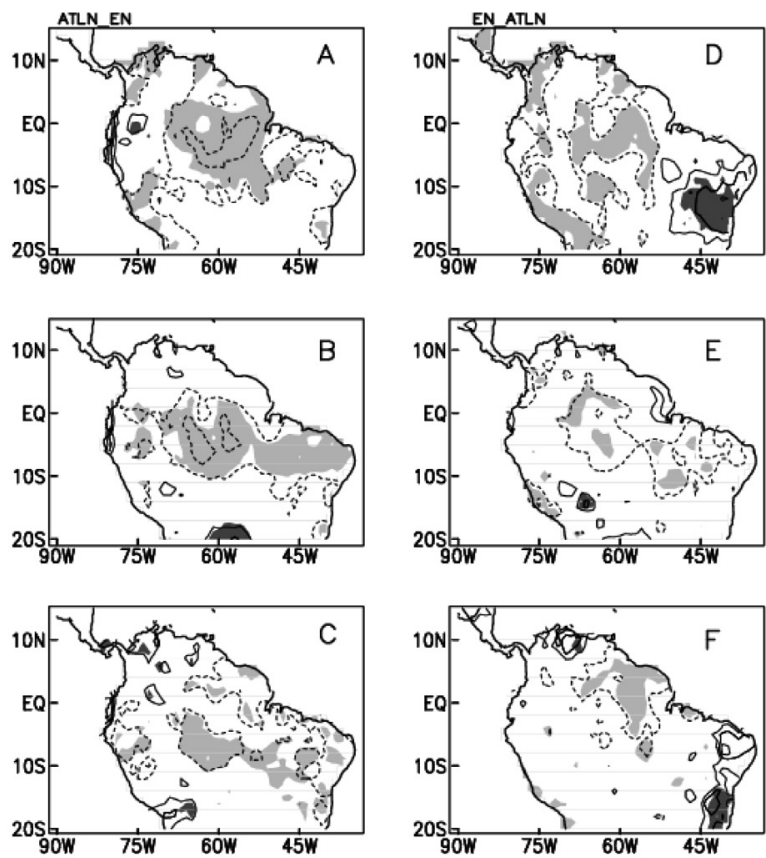

Figura 3 - Composições de anomalias normalizadas de precipitação para 0 período de: a) DJF (0), b) MAM (+1) e c) JJA (+1) para eventos ATLN_EN e d) DJF (0), e) MAM (+1) e f) JJA (+1) para eventos EN ATLN. Contornos contínuos (pontilhados) correspondem aos valores positivos (negativos). Intervalo de contorno é de 0.2. Sombreados em escala de cinza claro (escuro) definem regiões com valores negativos (positivos) significativos ao nível de confiança de $95 \%$.

nossas análises. Em MAM(+1), embora as ATSM persistam na parte central do Pacífico, o padrão de anomalias de PNM náo caracteriza o padrão de El Niño. Por outro lado, associado ao resfriamento da TSM no Atlântico equatorial um gradiente leste-oeste em PNM é estabelecido entre o Pacífico e Atlântico. No AT, consistente com os padróes de ATSM em MAM(+1) e JJA(+1), há sinais de um fraco dipolo inter-hemisférico negativo de anomalias de PNM.

Os campos correspondentes de anomalias de precipitação são apresentados na Figura 3 para os trimestres $\mathrm{DJF}(0)$, $\operatorname{MAM}(+1)$ e JJA(+1). As composiçóes do caso ATLN_EN são mostradas nas Figuras 3 a-c. Anomalias negativas significativas são encontradas em $\mathrm{DJF}(0)$ na regiáo nordeste do Brasil (NEB), na parte central da bacia Amazônica estendendo-se para leste e sobre as regióes da Venezuela, Guiana e Suriname. Anomalias negativas significativas também são notadas sobre o norte da Colômbia e sobre o sul do Peru. Para o trimestre MAM(+1) (Figura 3b), anomalias negativas significativas ainda persistem desde o norte do NEB até a região central da América do Sul e entre a linha do equador e $10^{\circ} \mathrm{S}$. Para o trimestre JJA $(+1)$ (Figura 3c), anomalias negativas significativas aparecem por toda a região sul da bacia Amazônica e em pequenas áreas das regióes sudeste da Amazônia, nordeste e costeira da América do Sul.
Em relação ao caso descrito acima, diferenças marcantes são notadas na composiçáo da precipitaçáo para o padrão EN_ATLN (Figuras $3 \mathrm{~d}$-f). Para o período de DJF(0) (Figura $3 \mathrm{~d}$ ), são observadas anomalias negativas significativas nas regiốes da Venezuela e Colômbia, nas partes central e sul da Bacia Amazônica, e anomalias positivas no sul do NEB, enquanto o centro de anomalia positiva sobre o Equador observado para o mesmo trimestre para o caso ATLN_EN desaparece totalmente. Para o período de MAM(+1) (Figura 3 e) o padrão de anomalias é similar ao padrão de $\operatorname{MAM}(+1)$ para o caso anterior, no entanto, anomalias significativas são notadas em pequenas áreas.

Para o caso EN_ATLN no trimestre JJA (+1) (Figura 3f), anomalias positivas de precipitação são notadas sobre o leste do NEB e norte da América do Sul, e anomalias negativas significativas, no nordeste e leste da bacia Amazônica, entre aproximadamente $10^{\circ} \mathrm{S}$ e $10^{\circ} \mathrm{N}$, que formam um corredor zonal bastante seco e tornam a estação seca ainda mais intensa nestas regióes.

\section{Fase negativa do ENOS e suas relações com a precipitação nas regiões norte e nordeste da América do Sul}

Os padróes espaciais de ATSM associados ao desenvolvimento do gradiente interbacias na fase inicial do evento La Nińa no Pacífico, ou seja, um aquecimento no Atlântico Equatorial na fase inicial do evento frio no Pacífico são apresentados na Figura 4 (painéis à esquerda). Esses padróes mostram o fortalecimento de um evento La Nińa com início em JJA(0). Nesse mesmo tempo, ATSM positivas estendem-se por toda a regiáo do Atlântico equatorial, definindo um gradiente interbacias de ATSM entre os oceanos Pacífico e Atlântico equatorial. Consistente com o padrão de TSM, um gradiente leste-oeste de anomalias de PNM (Figura 5, painéis à esquerda), com anomalias positivas no Pacífico tropical leste e negativas no Atlântico Equatorial, está bem estabelecido, favorecendo a intensificação da La Nińa. Esse gradiente leste-oeste em PNM persiste até JJA(+1), quando se enfraquece. Infere-se através deste padrão anômalo de PNM que se forma uma circulação de Walker, com subsidência no Pacífico central e leste e costa oeste da América do Sul e ascendência no restante da faixa tropical. A partir de DJF(0) anomalias negativas de TSM no Pacífico associadas à La Niña restringem-se a parte central da bacia. No AT, o resfriamento no ATN, em resposta à La Nińa, como mostrado em estudos anteriores (Enfield e Mayer 1997), ocorre no trimestre $\operatorname{MAM}(+1)$, e embora fraco, persiste até JJA $(+1)$.

O desenvolvimento do evento La Niña com gradiente interbacias notado durante a fase de decaimento do evento é apresentado na Figura 4 (painéis à direita, LN_ATLP). O evento La Nińa no Pacífico tem início a partir de JJA(0) e 


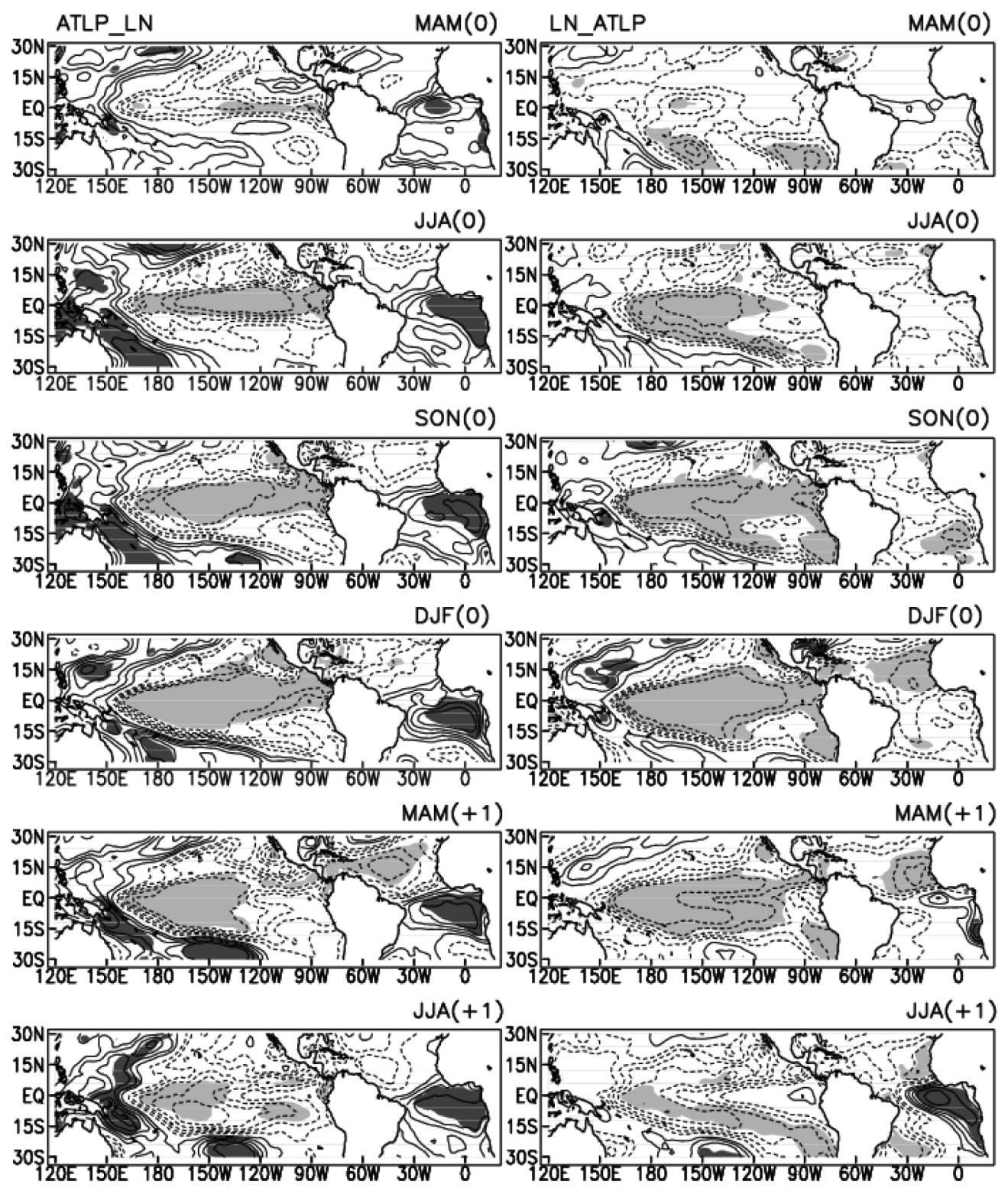

Figura 4 - Idem Figura 1, exceto para os eventos ATLP_LN e LN_ATLP.

atinge a máxima intensificação em $\mathrm{DJF}(0)$. Diferentemente do caso anterior, o gradiente leste-oeste de PNM entre o Pacífico e Attântico equatorial não é notado nesse período (Figura 5, painéis à direita). ATSM negativas no ATN surgem em resposta ao resfriamento do Pacífico na fase madura do evento de La Nińa em DJF(0). No ATS, ATSM positivas são observadas ao longo da costa oeste da África a partir de $\operatorname{MAM}(+1)$, quando se forma o gradiente zonal interbacias, com anomalias de sinais opostos no Pacífico central e Atlântico equatorial. Em resposta ao gradiente de ATSM, um gradiente leste-oeste de anomalias de PNM entre o Pacífico e Attântico se estabelece em $\operatorname{MAM}(+1)$. $\mathrm{O}$ gradiente interbacias não persiste em JJA(+1) devido à presença de ATSM fracas, mas positivas, no Pacífico leste equatorial. Vale notar que fracas ATSM negativas são notadas ao longo da costa leste do NEB em MAM(+1), que se intensificam em JJA(+1).

Composiçôes das anomalias de precipitação referentes aos eventos La Nińa mostrados na Figura 4 para os trimestres $\mathrm{DJF}(0), \operatorname{MAM}(+1)$ e JJA(+1) são apresentados nas Figuras 6 (a-c) e 6 (d-f), respectivamente. Para o caso ATLP_LN (Figuras $6 \mathrm{a}-\mathrm{c}$ ), anomalias positivas significativas são encontradas no trimestre $\mathrm{DJF}(0)$ no NEB (Figura 6a). Outros dois centros anômalos, porém, com pequenas áreas significativas também compóem esse campo, um positivo na regiáo de fronteira 

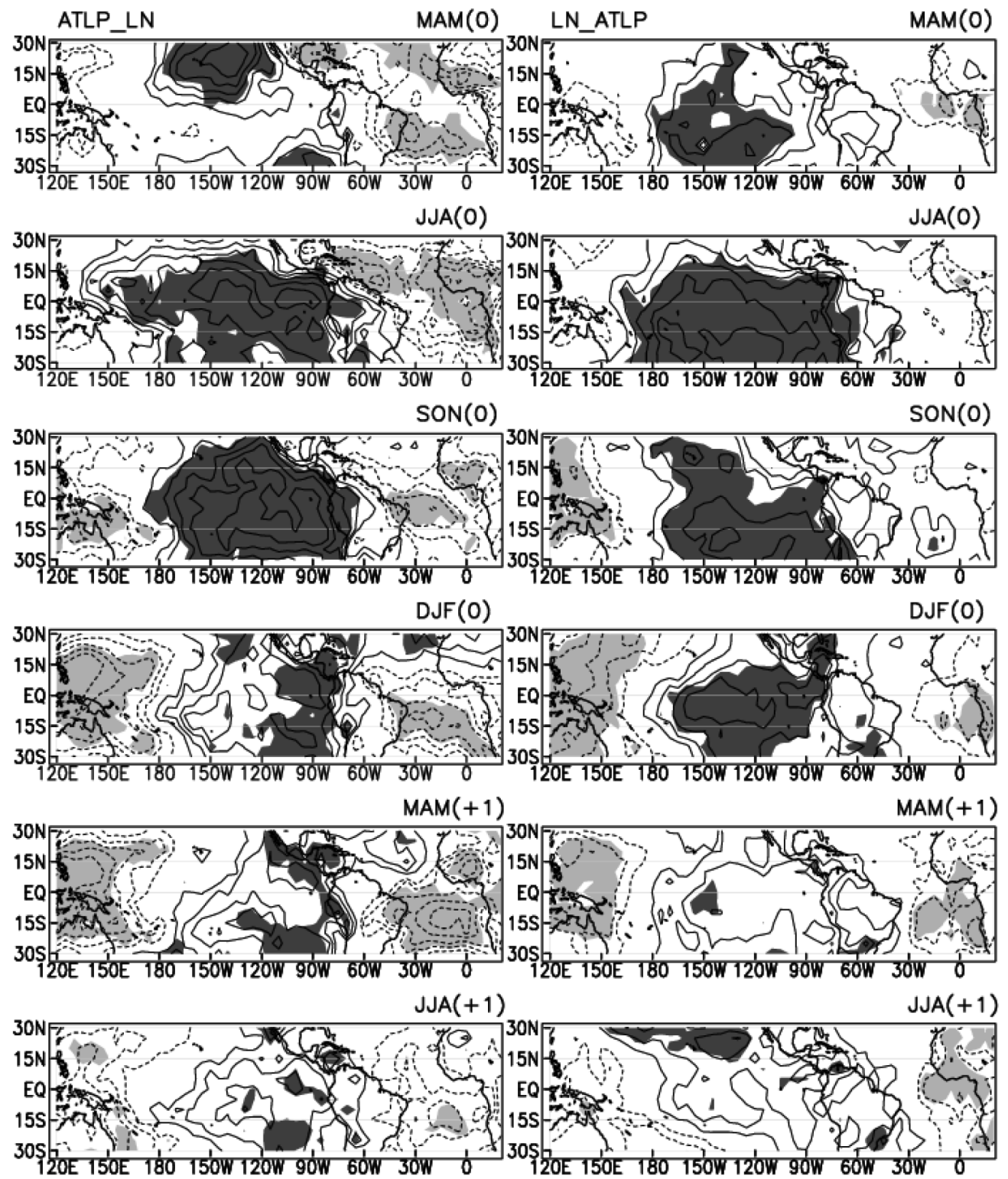

Figura 5 - Idem Figura 2, exceto para os eventos ATLP_LN e LN_ATLP.

entre Brasil, Colômbia e Venezuela e outro negativo no Sul do Equador e Leste do Peru. Para o trimestre MAM(+1) (Figura $6 \mathrm{~b})$, anomalias positivas significativas englobam a maior parte do NEB, e as no norte da América do Sul desaparecem e são substituídas por anomalias negativas, principalmente na região da fronteira da Colômbia com a Venezuela. Essas anomalias negativas contribuem para que a região norte da América do Sul se torne uma região seca nesse período.

No trimestre JJA $(+1$ ) (Figura 6c) as anomalias positivas persistem em parte do NEB e também se expandem em direção à região central da América do Sul definindo um centro de anomalias positivas sob as regióes centrais, leste e sul da bacia
Amazônica, porém com as áreas com anomalias significativas mais espalhadas do que nos trimestres anteriores. No extremo norte e oeste ainda são observadas anomalias negativas, porém, as anomalias significativas se limitam à porção norte da bacia Amazônica.

Para eventos LN_ATLP em DJF(0), anomalias positivas de precipitaçáo típicas de eventos de La Ninã aparecem em pequenas áreas espalhadas desde a regiáo costeira no norte do NEB ao longo da costa litorânea até extremo noroeste da Bacia Amazônica, porém as áreas com anomalias positivas significativas se limitam ao noroeste da América do Sul (Figura 6d). Para o trimestre MAM(+1) (Figura 6e), há quase uma 

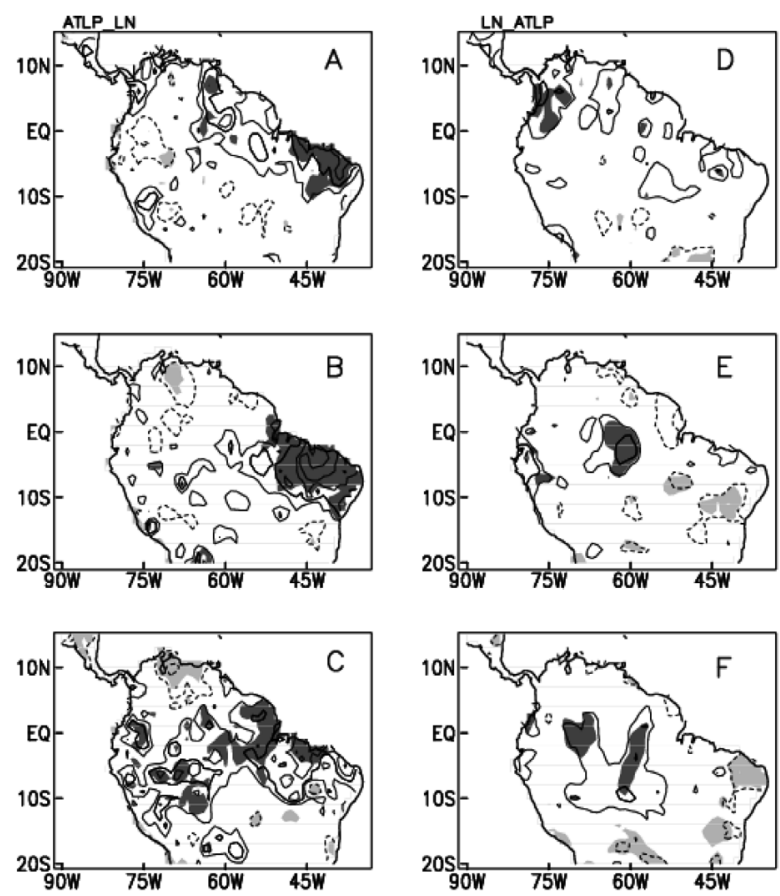

Figura 6 - Idem Figura 3, exceto para os eventos ATLP_LN e LN_ATLP.

reversão no sinal das anomalias por quase toda a região de estudo, de forma que anomalias positivas (negativas) surgem nas regiōes oeste, central e sul (norte e nordeste) da Bacia Amazônica. Também, anomalias negativas significativas são observadas no sul e leste do NEB. No trimestre JJA(+1) (Figura 6f), todos os centros anômalos significativos descritos no trimestre anterior se intensificam.

\section{DISCUSSÃO}

Diferenças marcantes são notadas na composiçẫo da precipitação para os padrôes EN_ATLN e ATLN_EN. Para o período de $\mathrm{DJF}(0)$ o padrão de precipitaçáo para o caso ATLN_EN é modulado por variaçôes que ocorrem nos oceanos Pacífico e Attântico equatorial desde JJA(0) associadas à circulação de Walker anômala inferida pelo campo de anomalias de PNM (Figura 2, painéis à esquerda) com ascendência na região do Pacífico leste e subsidência sobre a regiấo da América do Sul e Atlântico adjacente. Por outro lado, para o caso EN_ATLN as anomalias negativas de precipitaçáo são principalmente pelas ATSM que ocorrem na regiâo do Pacífico associadas ao El Niño, consistente com estudos anteriores dentre eles o de Marengo (1992), enquanto que as anomalias positivas de precipitação no NEB podem estar associadas à presença de ATSM positivas no ATS que persistem de $\mathrm{SON}(0)$ a JJA(+1).

Para os trimestres de MAM(+1) e JJA(+1) os padróes anômalos de precipitação podem estar associadas ao gradiente interbacias que persiste por todos os trimestres da evolução do padrão de anomalias ATLN_EN e também ao fraco gradiente inter-hemisférico positivo (negativo) de ATSM (de anomalias de PNM) (Figuras 1 e 2, painéis à esquerda). Por outro lado, o enfraquecimento das anomalias de precipitação para o período de $M A M(+1)$ durante o eventos EN_ATLN pode ser resultado do deslocamento das anomalias negativas de PNM para leste, que passam a ocupar a maior parte da regiáo norte da América do Sul, como pode ser visto na Figura 2 (painéis à direita). Em JJA(+1) para o caso EN_ATLN condiçóes secas na parte nordeste e leste da bacia estâo relacionadas às variaçóes de TSM no AT, com valores negativos no Attântico equatorial e ATS e positivos mas não significativos no ATN. Esse padrão anômalo de TSM no AT modula a posiçáo e intensidade da Zona de Convergência Intertropical (ZCIT) e, por conseguinte, a precipitação na América do Sul (Ronchail et al.2002), enquanto que, as anomalias positivas de precipitação no leste do NEB podem ser justificadas pela presença de ATSM positivas notadas ao longo da costa leste do NEB em JJA(+1).

Considerando os eventos La Niña, os padróes de precipitação durante eventos ATLP_LN para o período de $\mathrm{DJF}(0)$, podem estar relacionadas à variabilidade do Pacífico e Atlântico tropicais através dos gradientes interbacias e interhemisférico, ambos atuando no mesmo sentido. Por outro lado, para eventos LN_ATLP em DJF(0), anomalias positivas de precipitaçáo típicas de eventos de La Ninã aparecem em pequenas áreas espalhadas desde a regiáo costeira no norte do NEB ao longo da costa litorânea até extremo noroeste da Bacia Amazônica, porém as áreas com anomalias positivas significativas se limitam ao noroeste da América do Sul (Figura 6d). Consistente com esses resultados, Grimm et al. (1998) mostraram que a maior influência dos eventos de La Niña ocorre nas regióes nordeste da bacia Amazônica, porém, esses autores não descartaram a hipótese de existirem outras regiốes com anomalias positivas também consistentes, uma vez que $o$ número de estaçóes meteorológicas e a série de eventos de La Niña utilizado limitam a abrangência de seus resultados. Além disso, ressalta-se que o fato de as anomalias significativas serem restritas a pequenas áreas pode ser decorrente da variabilidade espacial das anomalias de precipitaçáo para os diferentes eventos selecionados na análise de composição. $\operatorname{Em~MAM(+1)~}$ e JJA(+1) anomalias positivas de precipitação notadas na regiâo central e sul da bacia Amazônica estão relacionadas à La Nińa. No entanto, as anomalias negativas de precipitação no NEB estão associadas às ATSM negativas que persistem ao longo da costa leste do NEB em MAM (+1) e JJA(+1). Consistente com resultados anteriores, a predominância de condiçôes anômalas no Atlântico equatorial e sul de mesmo sinal às do Pacífico, pode reverter o sinal da La Niña na precipitação na região nordeste da América do Sul (Giannini et al. 2004, Kayano e Andreoli 2006 e Andreoli e Kayano 2006). 


\section{CONCLUSÃO}

Os padróes de precipitação para os eventos ENOS para os quais o gradiente interbacias se forma em sua fase inicial apresentam anomalias mais acentuadas do que os eventos ENOS com o gradiente interbacias na sua fase de decaimento. Um aspecto que pode justificar é que no caso do MEA frio (ou quente) na fase inicial do El Nino (ou La Niña), um gradiente inter-hemisférico positivo (ou negativo) que se forma em $\mathrm{MAM}(+)$ e persiste até $\mathrm{JJA}(+1)$, colabora para fortalecer o efeito do El Niño (ou La Niña) na precipitação. Portanto ambos o gradiente interbacias formado pelo El Niño (ou La Niña) no Pacífico e MEA frio (ou quente) no Atlântico Equatorial, e o gradiente inter-hemisférico positivo (ou negativo) contribuem para o padrão seco (ou chuvoso) na maior parte do norte e nordeste da América do Sul do caso ATLN_EN (ou ATLP_LN).

Além disso, para os eventos ENOS que se desenvolvem sob condiçôes de normalidade ou mesmo sinal no AT, o impacto na precipitação é mais significativo na região norte e centro-oeste da bacia, associado ao fato de que nesses casos, o gradiente leste-oeste de PNM entre o Pacífico e Atlântico é menos intenso e deslocado para leste.

Portanto, um aspecto importante mostrado nessas análises é o papel do gradiente interbacias atuando juntamente com o gradiente inter-hemisférico para reforçar o padrão de precipitação. Esses resultados têm aplicabilidade para propósitos de monitoramento climático.

\section{AGRADECIMENTOS}

Os autores agradecem o Conselho Nacional de Desenvolvimento Científico e Tecnológico (CNPq/Processo 569749/2008-5) e a Financiadora de Estudos e Projetos (FINEP/Projeto REMCLAM), pelo apoio financeiro à pesquisa e aos três revisores anônimos pelos seus comentários e sugestóes.

\section{BIBLIOGRAFIA CITADA}

Aceituno, P. 1988. On the functioning of the Southern Oscillation in the South American sector: Part I. surface climate. Monthly Weather Review, 166: 505-524.

Allan, R.; Ansell, T. 2006. A new globally completed monthly historical gridded mean sea level pressure dataset (HadSLP2): 1850-2004. Journal of Climate, 19: 5816-5842.

Andreoli, R.V.; Kayano, M.T. 2006. Tropical Pacific and South Atlantic effects on rainfall variability over northeastern Brazil. International Journal of Climatology, 26: 1895-1912.

Andreoli, R.V.; Souza, R.A.F.; Kayano, M.T.; Candido, L.A. 2012. Seasonal anomalous rainfall in the central and eastern Amazon and associated anomalous oceanic and atmospheric patterns. International Journal of Climatology, 32: 1193-1205, DOI: $10.1002 /$ joc. 2345 .
Beck, C.; Grieser, J.; Rudolf, B. 2005. A new monthly precipitation climatology for the global land areas for the period 1951 to 2000. Climate Status Report, Ger. Weather Serv., Offenbach, Germany.

Curtis, S.; Hastenrath, S. 1995. Forcing of anomalous sea surface temperature evolution in the tropical Atlantic during Pacific warm events. Journal of Geophysical Research, 100: 15835-15847.

Enfield, D.B.; Mayer, D.A. 1997. Tropical Atlantic sea surface temperature variability and its relation to El Niño-Southern Oscillation. Journal of Geophysical Research, 102: 929-945.

Giannini, A.; Chiang, J.C.H.; Cane, M.A.; Kushnir, Y.; Seager, R. 2001. The ENSO teleconnection to the tropical Atlantic Ocean: contributions of the remote and local SSTs to rainfall variability in the tropical Americas. Journal of Climate, 14: 4530-4544.

Giannini, A.; Saravanan, R.; Chang, P. 2004. The preconditioning role of Tropical Atlantic variability in the development of the ENSO teleconnection: implications for the prediction of Nordeste rainfall. Climate Dynamics, 22: 839-855, DOI: 10.1007/s00382-004-0420-2.

Grimm, A.M.; Ferraz, S.E.T.; Gomes, J. 1998. Precipitation anomalies in southern Brazil associated with El Niño and La Niña events. Journal of Climate, 11: 2863-2880.

Grimm, A.M.; Barros, V.R.; Doyle, M.E. 2000. Climate variability in southern South America associated with El Niño and La Niña events. Journal of Climate, 13: 35-58.

Grimm, A.M. 2004. How do La Niña events disturb the summer monsoon system in Brazil? Climate Dynamics, 22: 123-138.

Jury, M.R. 2009. An interdecadal American rainfall mode. Journal of Geophysical Research, 114: D08123.

Kayano, M.T.; Moura, A.D. 1986. O El Nino de 1982-83 e a precipitação sobre a América do Sul. Revista Brasileira de Geofisica, 4: 201-214.

Kayano, M.T; Andreoli, R.V. 2006. Relationships between rainfall anomalies over northeastern Brazil and the El Nin o Southern Oscillation. Journal of Geophysical Research, 111: D13101.

Kayano, M.T.; Andreoli, R.V.; Souza, R.A.F. 2011. Evolving anomalous SST patterns leading to ENSO extremes: relations between the tropical Pacific and Atlantic Oceans and the influence on the South American rainfall. International Journal of Climatology, 31: 1119-1134.

Latif, M.; Grötzner, A. 2000. On the equatorial Atlantic oscillation and its response to ENSO. Climate Dynamics, 16: 213-218.

Liebmann, B.; Marengo, J.A. 2001. Interannual variability of the rainy season and rainfall in the Brazilian Amazon Basin. Journal of Climate, 14:4308-4318

Melice, J.L.; Servain, J. 2003. The tropical Atlantic meridional SST gradient index and its relationships with the SOI, NAO and Southern Ocean. Climate Dynnmics, 20: 447-464.

Marengo, J.A. 1992. Interannual variability of surface climate in the Amazon basin. International Journal of Climatology, 12: 853-863.

Marengo, J.A. 2006. On the hydrological cycle of the Amazon basin: a historical review and current state-of-the-art. Revista Brasileira de Meteorologia, 21: 1-19.

Marengo, J.A.; Nobre, C.A.; Tomasella. J.; Oyama, M.D.; Sampaio, G.; Camargo, H.; Alves, L.M. 2008. The drought of Amazonia in 2005. Journal of Climate, 21: 495-516. 
Ronchail, J.; Cochonneau, G.; Molinier, M.; Guyot, J.L.; Chaves, A.G.D.; Guimaraes, V.; de Oliveira, E. 2002. Interannual rainfall variability in the Amazon basin and sea-surface temperatures in the equatorial Pacific and the tropical Atlantic Oceans. International Journal of Climatology, 22: 1663-1686.

Rodrígues-Fonseca, B.; Polo, I.; Garcia-Serrano, J.; Losada, T.; Mohino, E.; Mechoso, C.R.; Kucharski, F. 2009. Are Atlantic Niños enhancing Pacific ENSO events in recent decades? Geophysical Research Letters, 36: L20705, DOI: 10.1029/2009GL040048.

Rudolf, B., Rubel, F. 2005. Global precipitation, in Observed Global Climate, New Series on Landolt-Bornstein: Numerical Data and Functional Relationships in Science and Technology, vol. 6, edited by M. Hantel, p. 11.1-11.24, Springer, Berlin.
Smith, T.M.; Reynolds, R.W.; Peterson, T.C.; Lawrimore, J. 2008. Improvements to NOAA's historical merged land-ocean surface temperature analysis (1880-2006). Journal of Climate 21: 2283-2296, DOI: 10.1175/2007JCLI2100.1.

Trenberth, K.E. 1997. The Definition of El Niño. Bulletin of the American Meteorological Society, 78: 2771-2777.

Wang, C. 2006. An overlooked feature of tropical climate: InterPacific-Atlantic variability. Geophysical Research Letters, 33: L12702, DOI: 10.1029/2006GL026324.

Yoon, J.-H.; Zeng, N. 2010. An Atlantic influence on Amazon rainfall. Climate Dynamics 34: 249-264. DOI 10.1007/s00382009-0551-6.

Zebiak, S.E. 1993. Air-sea interaction in the equatorial Atlantic region. Journal of Climate, 6: 1567-1586.

Recebido em: 19/07/2012

Aceito em: 04/04/2013 